\title{
Cardiovascular responses to plyometric exercise are affected by workload in athletes
}

\author{
Hamid Arazi ${ }^{1}$, Abbas Asadi ${ }^{2}$, Seyed Amir Mahdavi ${ }^{1}$, Seyed Omid Mirfalah Nasiri ${ }^{1}$ \\ ${ }^{1}$ Department of Exercise Physiology, Faculty of Sport Sciences, University of Guilan, Rasht, Iran \\ ${ }^{2}$ Young Researchers and Elite Club, Roudbar Branch, Islamic Azad University, Roudbar, Iran
}

Postep Kardiol Inter 2014; 10, 1 (35): 2-6

DOI: $10.5114 /$ pwki.2014.41458

\begin{abstract}
A bstract
Introduction: With regard to blood pressure responses to plyometric exercise and decreasing blood pressure after exercise (post-exercise hypotension), the influence of different workloads of plyometric exercise on blood pressure is not clear.

Aim: The purpose of this investigation was to examine the effects of a low, moderate and high workload of plyometric exercise on the post-exercise systolic (SBP) and diastolic blood pressure (DBP), heart rate (HR) and rate-pressure product (RPP) responses in athletes.

Material and methods: Ten male athletes (age: $22.6 \pm 0.5$ years; height: $178.2 \pm 3.3 \mathrm{~cm}$; and body mass: $75.2 \pm 2.8 \mathrm{~kg}$ ) underwent $\mathrm{PE}$ protocols involving $5 \times 10$ reps (Low Workload - LW), $10 \times 10$ reps (Moderate Workload - MW), and $15 \times 10$ reps (High Workload - HW) depth jump exercise from a 50-cm box in 3 non-consecutive days. After each exercise session, SBP, DBP and HR were measured every $10 \mathrm{~min}$ for a period of $70 \mathrm{~min}$.

Results: No significant differences were observed among post-exercise SBP and DBP when the protocols (LW, MW and HW) were compared. The MW and HW protocols showed greater increases in HR compared with LW. Also the HW indicated greater increases than LW in RPP at post-exercise $(p<0.05)$.

Conclusions: All protocols increased SBP, HR and RPP responses at the $10^{\text {th }}$ and $20^{\text {th }}$ min of post-exercise. With regard to different workloads of plyometric exercise, HW condition indicated greater increases in HR and RPP and strength and conditioning professionals and athletes must keep in their mind that HW of plyometric exercise induces greater cardiovascular responses.
\end{abstract}

Key words: plyometric, systolic blood pressure, diastolic blood pressure, heart rate, different workload.

\section{Introduction}

Recently, attention has been focused not only on the cardiovascular benefits of physical training, but also on the effects of an acute exercise session. After an acute exercise bout, blood pressure (BP) levels are reduced for minutes or hours in relation to pre-exercise levels [1]. This phenomenon is called post-exercise hypotension (PEH) and has been widely investigated because of its importance for the treatment and prevention of arterial hypertension [1].

Endurance and resistance exercise/training has also been recommended as part of a comprehensive exercise program to reduce cardiovascular risk in the general population [2] and hypertensives [3] and also is the main exercise for the development of physical performance [4]. Nowadays, plyometric exercise/training is used in the conditioning schedule by athletes and condition- ing coaches for improving muscular performance [5], but evidence of the effects of this exercise on PEH is scarce. Some evidence suggests that endurance and resistance exercise favorably alters resting arterial BP in persons with hypertension [6-8], whereas it is relatively unknown whether plyometric exercise $(\mathrm{PE})$ results in a $\mathrm{PEH}$ response.

According to the important role of $\mathrm{PE}$, the information about this type of exercise on cardiovascular variables, especially on BP, is not completely understood. Only a few studies have investigated the BP responses following $P E$, and these have shown conflicting results [9-11]. Previous studies reported that PE can increase BP after each set of exercise $[10,11]$, but the information about the effects of PE on PEH is scarce and no study has examined this approach.

\section{Corresponding author:}

Abbas Asadi MSc, Young Researchers and Elite Club, Roudbar Branch, Islamic Azad University, Imam Khomeini St, 446151146 Roudbar, Iran, phone: 989113213041, e-mail: abbas_asadi1175@yahoo.com

Received: 28.12.2013, accepted: 9.01.2014. 
Since designing plyometric exercise can depend on 5 acute variables - choice of exercise, rest interval, intensity, number of sets and repetitions (workload) - more knowledge is required about the effects of these characteristics on PEH, especially the workload of the bout. Because BP responses during the recovery period can be influenced by exercise workload, it is possible that different PE workload may also have distinct effects on $\mathrm{PEH}$. However, there is scarce information about the influence of exercise workload on BP; only a few studies have examined the BP and heart rate (HR) responses to different exercise workload and they found conflicting results [1214]. Also, although a few researchers have investigated the effects of different resistance or endurance exercise workloads on BP and HR [12-14], limited data exist regarding the effects of different workloads of $\mathrm{PE}$ on $\mathrm{PEH}$ and $\mathrm{HR}$ responses.

\section{Aim}

Thus, this study investigated the influence of a session of PE with differing workload on responses of diastolic BP (DBP), HR and systolic BP (SBP) and, consequently, of the rate-pressure product (RPP) in male athletes.

\section{Material and methods}

The participants underwent 3 random protocols of exercise, in an order defined in a counterbalanced way. Each session took place on the same day, with a total of 3 other visits to the laboratory, with intervals of 1 week in between. At least a week prior to treatments, participants were admitted to the laboratory and age, body mass and height were recorded. During this session, each participant was instructed in the proper form and technique of depth jump exercise (familiarization session). Treatment sessions were initiated between 2:00 and 4:00 PM.

The subjects were instructed to observe the following recommendations before the exercise sessions: a) take a light meal $2 \mathrm{~h}$ before the experiments; $\mathrm{b}$ ) not to do any type of physical activity within $48 \mathrm{~h}$ before the test; c) abstain from alcohol, caffeinated drinks or stimulants for $24 \mathrm{~h}$; d) make as little effort as possible during the trip to the laboratory. All subjects performed the following procedures: a) Low Workload (LW) plyometric exercise (5 $\times 10$ rep), b) Moderate Workload (MW) plyometric exercise $(10 \times 10$ rep), and c) High Workload (HW) plyometric exercise $(15 \times 10$ rep). Before performing the protocols, the participants performed 10 min warm-up, and then remained seated for $10 \mathrm{~min}$, in a calm and quiet environment. Then, the HR and BP were measured at rest. Then they moved to the exercise room, where they performed $\mathrm{PE}$. The ambient temperature was fixed at $27 \pm 1^{\circ} \mathrm{C}$ and the air humidity during the tests ranged between $60 \%$ and $70 \%$. After completing the exercise protocols, participants returned to the laboratory and were seated for $70 \mathrm{~min}$ in a quiet and comfortable place, to measure the post-exercise BP and HR every 10 min (post-intervention period). The cardiovascular variables were measured by using the same equipment and experienced appraiser at rest and after exercise for all participants.

Ten college-aged men (age: $22.6 \pm 0.5$ years; height: $178.2 \pm 3.3 \mathrm{~cm}$; and body mass, $75.2 \pm 2.8 \mathrm{~kg}$ ) volunteered to participate in this study. All subjects were athletes (college level) and trained at least 3 times a week for $90 \mathrm{~min}$. The following exclusion criteria were observed: a) use of drugs that could influence cardiovascular responses at rest or during exercise, b) musculoskeletal limitations that would cause the exercises to be contraindicated, and c) diagnosis of hypertension, heart disease or other cardiovascular problem that would cause the exercises to be contraindicated. All risks associated with the experimental procedures were explained prior to involvement in the study and each participant was asked to complete a written informed consent form and a medical health questionnaire before assessment. Procedures and the study were conducted in accordance with ethical standards in sport and exercise science research [15] and approved by the Local Ethics Committee.

After a 10 min standard warm-up (including light running, static stretching and ballistic movements), participants performed PE protocols. Participants in LW performed 5 sets of 10 repetitions of depth jump from a 50$\mathrm{cm}$ high box, and participants in MW performed 10 sets of 10 repetitions of depth jump, whereas participants in HW performed 15 sets of 10 repetitions of depth jump onto a wrestling-type mat. The duration of rest intervals in between jumps and sets was $8 \mathrm{~s}$ and $120 \mathrm{~s}$, respectively. These exercise programs were based on recommendations of intensity from Chu [16] and Ebben et al. [17].

Systolic blood pressure and DBP were measured on the left upper arm by the auscultation method using a sphygmomanometer (Missouri ${ }^{\circledR}$ ) and a stethoscope (Rappaport $^{\oplus}$ GF Health Products, Northeast Parkway Atlanta). An evaluator assessed the SBP (first Korotkoff sound) via auscultation and the DBP (fifth Korotkoff sound) at rest and after PE protocols for every $10 \mathrm{~min}$. The measurement procedure was in accordance with the recommendations of the American Heart Association [18] and was conducted by an experienced evaluator. The HR was measured using a Polar S610i heart rate monitor (FIN, 90440, FINLAND) (bpm). During the measurements, the volunteers remained seated on a comfortable couch in an environment without noise. The rate-pressure product (RPP) was calculated as SBP $\times$ heart rate $(\mathrm{mm} \mathrm{Hg} \times \mathrm{bpm})$, as it is considered a reliable predictor of myocardial oxygen demand [19].

\section{Statistical analysis}

Data are presented as mean \pm standard deviation. A 2-way repeated-measures ANOVA followed by the Bonferroni post hoc test, where indicated, was used to ana- 
lyze SBP, DBP, HR and RPP. The level of significance was set at $p<0.05$ for all statistical procedures. All analyses were conducted using SPSS version 16.0 (SPSS Inc., Chicago, IL, USA).

\section{Results}

No significant differences were found among post-exercise SBP and DBP when the programs (LW, MW and HW) were compared. All workloads showed significant increases in the post-exercise SBP level at the $10^{\text {th }}$ and $20^{\text {th }}$ min of post-exercise, whereas HW showed significant increases in the SBP until the $50^{\text {th }}$ min of post-exercise (Figure 1). In DBP, no significant changes were observed for LW, whereas significant increases were seen at the $10^{\text {th }}$ min of post-exercise for MW and HW (Figure 2). Also, the HW protocol maintained significant increases in the post-exercise DBP until the $20^{\text {th }}$ min of post-exercise.

There was a significant group by time interaction which indicated significantly greater $\mathrm{HR}$ in $\mathrm{MW}$ and $\mathrm{HW}$ compared to LW at the $20-40^{\text {th }}$ min of post-exercise $\left(F_{5.4,57.6 \mathrm{GG}}=3.70, p<0.05\right)$. All workloads increased $\mathrm{HR}$ at the $10^{\text {th }} \mathrm{min}$ of post-exercise, whereas these increases were greater for MW and HW and remained until the $40^{\text {th }}$ min of post-exercise. Only HW showed a maintained elevation in HR until the $50^{\text {th }}$ min post-exercise (Figure 3).

There was a significant group by time interaction which indicated significantly greater RPP in HW compared to LW at the $30-50^{\text {th }}$ min of post-exercise $\left(F_{5.3,55.8 \mathrm{GG}}\right.$ $=3.50, p<0.05)$. All workloads showed increases in RPP at the $10^{\text {th }}$ and $20^{\text {th }}$ min of post-exercise, whereas these increases were greater for $\mathrm{MW}$ and $\mathrm{HW}$ and remained until the $40^{\text {th }}$ min of post-exercise. Only HW showed maintained elevation in RPP until the $50^{\text {th }}$ min post-exercise (Figure 4).

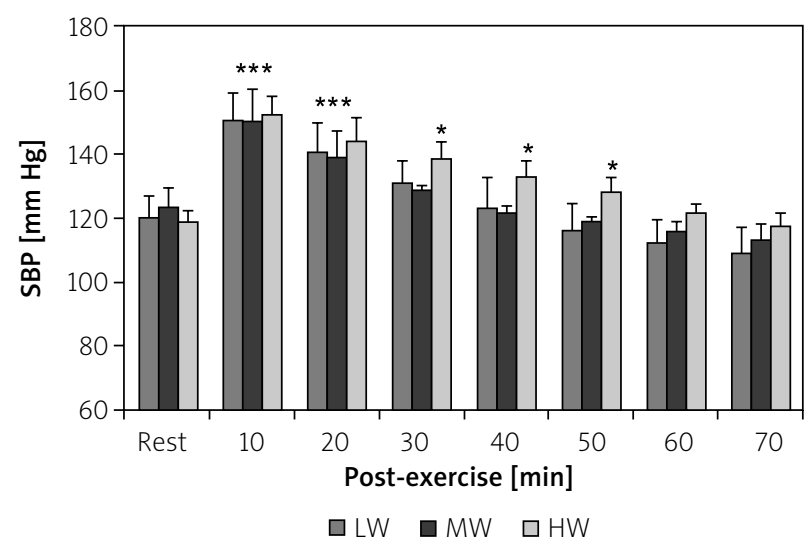

Figure 1. Mean \pm SD of systolic blood pressure (SBP) at rest and after performing low workload (LW), moderate workload (MW) and high workload (HW) plyometric exercise

*Significant changes $(p<0.05)$ compared with rest value

\section{Discussion}

With regard to the key role of plyometric exercise/ training for the development of muscular performance, the effects of this type of exercise on cardiovascular responses is important but few studies have focused on this area and information about this aspect is scarce. Therefore, the current investigation was designed to examine the influence of a session of PE with differing workload on PEH, HR and RPP. In this research, we found that post-exercise SBP was significantly enhanced by all workloads during 20 min of post-exercise, and these increases remained until the $50^{\text {th }}$ min of post-exercise for the HW group. Likewise, DBP was significantly increased in the $10^{\text {th }}$ min of post-exercise for both the MW and HW groups, whereas HW showed maintained elevation until the $20^{\text {th }}$ min of post-exercise.

Our results indicated that the workload can also independently influence the responses of SBP and DBP. The findings of the present study about SBP are in line with previous studies [12-14] while those concerning DBP are not in agreement with previous studies that investigated PEH after resistance and endurance exercises [12-14]. The BP increases when the exercise workload increases. For example, when subjects performed different workloads of resistance exercise (identified as light, moderate, and high), blood pressure was the highest for the high workload [14]. There is disagreement about the DBP response to resistance exercise; some authors report an increase and others report no change [12-14]. These discrepancies may reflect differences in measurement techniques (namely, auscultation and intra-arterial assessment) and timing of the measurement. The hemodynamic responses to muscular work in PE can also be linked to increased sympathetic activity and decreased

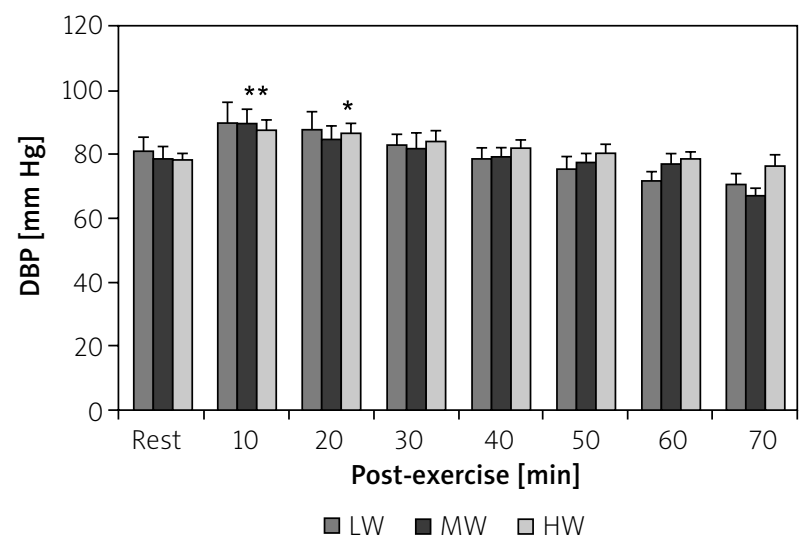

Figure 2. Mean \pm SD of diastolic blood pressure (DBP) at rest and after performing low workload (LW), moderate workload (MW) and high workload (HW) plyometric exercise

*Significant changes $(p<0.05)$ compared with rest value 


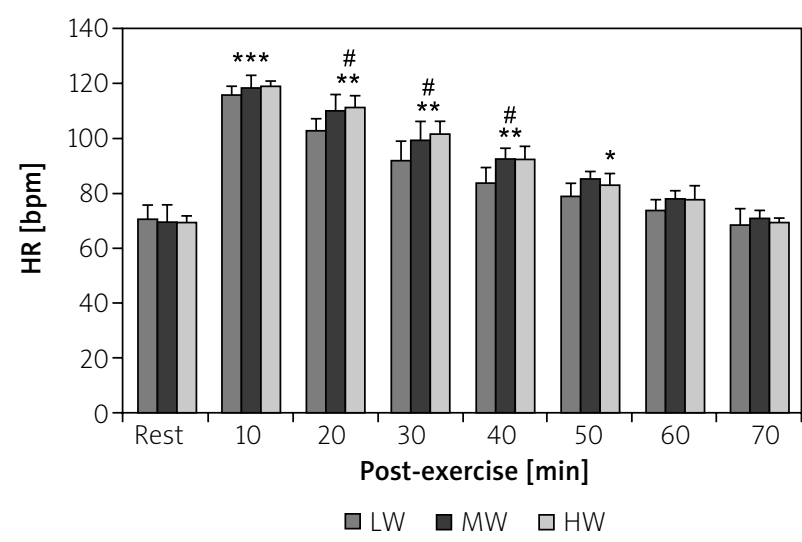

Figure 3. Mean \pm SD of heart rate $(H R)$ at rest and after performing low workload (LW), moderate workload (MW) and high workload (HW) plyometric exercise

*Significant changes $(p<0.05)$ compared with rest value, "significant changes $(p<0.05)$ between MW and HW with $L W$ value

parasympathetic activity, due to the greater activation of central command and muscle and joint mechanoreceptors, resulting in increases in BP [20]. The central mechanism involves the transmission of impulses from the motor cortex to the cardiovascular control center. On the other hand, the peripheral mechanism consists of a reflex pathway with multiple control bases [19, 21]. Finally, the increase in blood pressure could also be influenced by the number of motor units requested. In this case, muscle and joint mechanoreceptors, sensitive to the increase in voluntary strength (recruitment of motor units with increasing workload) and the load on the joints, inform the cardiovascular control center about the need to modify the cardiovascular responses to regulate the flow [22]. It seems that these mechanisms are common reasons for the enhancement of BP and more than for HW and resulting greater increases in $\mathrm{BP}$ following $\mathrm{PE}$.

In HR, all workloads showed significant increases at the $10^{\text {th }}$ min after $\mathrm{PE}$, and $\mathrm{MW}$ and $\mathrm{HW}$ remained elevated until the $40^{\text {th }}$ min of post-exercise, whereas this elevation remained until the $50^{\text {th }}$ min of post-exercise for HW. Also, MW and HW showed greater increases than LW at the 20$40^{\text {th }}$ min of post-exercise in HR. Our findings indicated that the workload can also play an important role for the response of HR. With increasing PE workloads, the response of HR increased. It seems that muscle metabolites and heat accumulation are directly related to exercise intensity, and the sweating rate is greater during PE, and with increasing workload these mechanisms were enhanced with a consequent elevation in HR [23]. In addition, the increased local muscle metabolite and/or heat production are also potential stimuli for the increases in $\mathrm{HR}$ after $\mathrm{PE}$, and these increases were higher for greater workloads [24, 25]. On the other hand, a decrease in muscle cell $\mathrm{pH}$ following PE may stimulate chemo-sensitive afferent fibers, thereby elevating HR [26]. The greater involvement of the fast-twitch

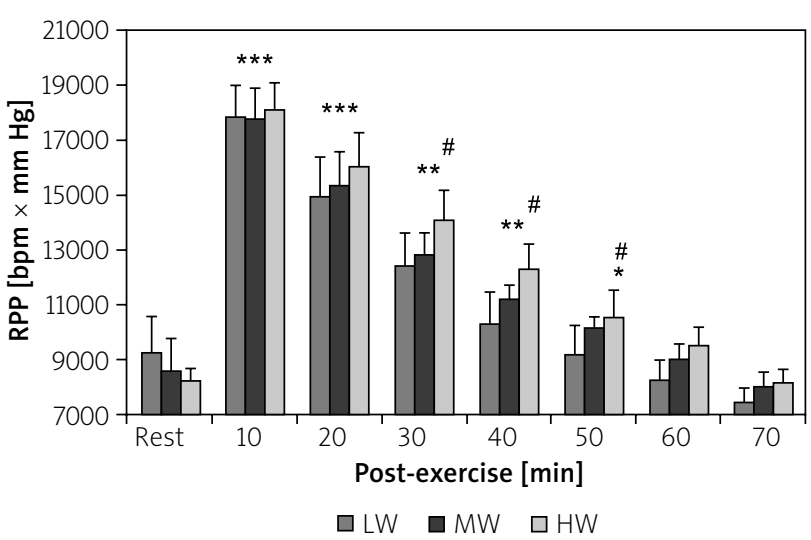

Figure 4. Mean \pm SD of rate-pressure product (RPP) at rest and after performing low workload (LW), moderate workload (MW) and high workload (HW) plyometric exercise

*Significant changes $(p<0.05)$ compared with rest value,

"significant changes $(p<0.05)$ compared with $L W$ value

muscle fibers and size of activated muscle mass may also stimulate increases in HR [27].

The RPP is regarded as an important noninvasive means of estimating myocardial oxygen demand.

In the current study we found increases in RPP for all of the workloads at the $10^{\text {th }}$ and $20^{\text {th }}$ min of post-exercise, and $\mathrm{MW}$ and $\mathrm{HW}$ remained elevated until the $40^{\text {th }}$ min of post-exercise, whereas this elevation remained until the $50^{\text {th }}$ min of post-exercise for HW. Also, HW showed greater increases than LW at the $30-40^{\text {th }}$ min of post-exercise in RPP. The results of this study suggest that with increased exercise workload, the myocardial oxygen increases. The mechanism(s) of this increase may be coupled increases in HR and SBP [19].

\section{Conclusions}

With regard to the results of this study the possible mechanism(s) that increases in BP and HR could be alterations in sympathetic nervous system function and vasculature responsiveness. Also, increase in $\mathrm{pH}$, muscle metabolites and heat production after plyometric exercise could be other mechanisms for these responses. It seems that these increases are greater for $\mathrm{HW}$, resulting in greater increases in HR and BP. Although the current study was the first to examine $\mathrm{PEH}$ following different workloads of PE, more studies are needed to define the role of $P E$ in altering cardiovascular variables in human subjects.

\section{References}

1. Kenny MJ, Seals DR. Postexercise hypotension. Key features, mechanisms and clinical significant. Hypertension 1993; 22: 653-664.

2. Piepoli M, Coats AJ, Adamopoulos S, et al. Persistent peripheral vasodilation and sympathetic activity in hypotension after maximal exercise. J Appl Physiol 1993; 75: 1807-1814. 
3. American College of Sports Medicine. ACSM's guidelines for exercise testing and prescription. Lippincott Williams and Wilkins, Philadelphia 2000.

4. Arazi H, Faraji H, Ghahremani Moghadam M, Samadi A. Effects of concurrent exercise protocols on strength, aerobic power, flexibility and body composition. Kinesiology 2011; 43: 155-162.

5. Arazi H, Coetzee B, Asadi A. Comparative effect of land and aquatic based plyometric training on the jumping ability and agility of young basketball players. South African J Res Sport Phys Edu Rec 2012; 34: 1-14.

6. Forjaz CL, Cardoso CG JR, Rezk CC, et al. Postexercise hypotension and hemodynamics: the role of exercise intensity. J Sports Med Phys Fitness 2004; 44: 54-62.

7. Pescatello LS, Franklin BA, Fagard R, et al. American College of Sports Medicine position stand. Exercise and hypertension. Med Sci Sports Exerc 2004; 36: 533-553.

8. O'Connor PJ, Bryant CX, Veltri JP, Gebhardt SM. State anxiety and ambulatory blood pressure following resistance exercise in females. Med Sci Sports Exerc 1993; 25: 516-521.

9. Roltsch MH, Mendez T, Wilund KR, Hagberg JM. Acute resistive exercise does not affect ambulatory blood pressure in young men and women. Med Sci Sports Exerc 2001; 33: 881-886.

10. Arazi H, Asadi A, Nasehi M, Delpasand A. Cardiovascular and blood lactate responses to acute plyometric exercise in female volleyball and handball players. Sport Sci Health 2012; 8: 23-29.

11. Brown GA, Ray MW, Abbey BM, et al. Oxygen consumption, heart rate, and blood lactate responses to an acute bout of plyometric depth jumps in college-aged men and women. J Strength Cond Res 2010; 24: 2475-2482.

12. Fleck SJ. Cardiovascular adaptations to resistance training. Med Sci Sports Exerc 1988; 20: S146-S151.

13. Fleck SJ, Dean LS. Resistance-training experience and the pressor response during resistance exercise. J Appl Physiol 1987; 63: 116-120.

14. Wescott W, Howes B. Blood pressure response during weight training exercise. Nat Strength Cond Asso J 1983; 5: 67-71.

15. Harriss DJ, Atkinson G. Ethical standards in sport and exercise science research. Int J Sports Med 2011; 32: 819-821.

16. Chu DA. Jumping Into Plyometrics. Human Kinetics, Champaign 1998.

17. Ebben WP. Practical guidelines for plyometric intensity. Nat Strength Cond Asso 2009; 6: 12-16.

18. American Heart Association. Medical statements, exercise standards: a statement for health professionals from the American Heart Association. Circulation 1990; 82: 2286-2322.

19. Arazi H, Rahmaninia F, Hosseini K, Asadi A. Resting hormonal and cardiovascular responses to short term creatine supplementation and resistance exercises. Sci Sport 2014; in press.

20. Rowell LB, O'Leary DS. Reflex control of the circulation during exercise: chemo-reflexes and mechanoreflexes. J Appl Physiol 1990; 69: 407-418.

21. Carrington CA, Ubolsakka C, White MJ. Interaction between muscle metaboreflex and mechanoreflex modulation of arterial baroreflex sensitivity in exercise. J Appl Physiol 2003; 95: 43-48.

22. Prabhakar NR, Peng YJ. Peripheral chemoreceptors in health and disease. J Appl Physiol 2004; 96: 359-366.

23. Kawano $\mathrm{H}$, Nakagawa $\mathrm{H}$, Onodera $\mathrm{S}$, et al. Attenuated increases in blood pressure by dynamic resistance exercise in middle-aged men. Hypertension Res 2008; 31: 1045-1053.
24. Middlekauff HR, Nitzsche EU, Nguyen AH, Hoh CK. Modulation of renal cortical blood flow during static exercise in humans. Circulation Res 1997; 80: 62-68.

25. Halliwill JR, Taylor JA, Eckberg DL. Impaired sympathetic vascular regulation in humans after acute dynamic exercise. J Physiol 1996; 495: 279-288.

26. Victor RG, Bertocci LA, Pryor SL, Nunally RL. Sympathetic discharge is coupled to muscle cell pH during exercise in humans. J Clin Invest 1988; 82: 1301-1305.

27. Rozenek R, Rosenau L, Rosenau P, Stone MH. The effect of intensity on heart rate and blood lactate response to resistance exercise. J Strength Cond Res 1993; 7: 51-54. 